\title{
El miedo en los informativos de televisión
}

\author{
Jesús María OsÉs GoRRAIZ \\ Universidad Pública de Navarra \\ oses@unavarra.es \\ Aritz Bermejo AyestarÁn \\ Universidad Pública de Navarra \\ aritzbermejo@yahoo.es
}

Recibido: $26 / 01 / 2013$

Aceptado: 16/09/2013

\begin{abstract}
Resumen
En este trabajo se han analizado veintiún informativos, siete por cada uno de los principales canales de televisión españoles (TVE 1, Telecinco y Antena 3), al objeto de medir su contribución a la alarma social o clima de miedo que existe en el país y establecer, mediante la comparación de los resultados obtenidos, las similitudes y diferencias que existen entre la cadena pública y las privadas. Se ha atendido a diversos aspectos, como el nivel de miedo de los contenidos, el área temática de las informaciones y el momento temporal -pasado o futuro- al que se refieren las noticias y los titulares. La conclusión principal es que no se puede fijar una clara línea de separación entre el ente público y los canales comerciales. Palabras clave: miedo, informativos, televisión
\end{abstract}

\section{Fear in news programmes}

\begin{abstract}
In this research there have been analyzed twenty-one news programmes, seven per each of the major Spanish television channels (TVE 1, Telecinco and Antena 3). The aim of this work is to consider how these programmes contribute to the social alarm or to the climate of fear that nowadays exists in our country and, so, establish, by comparing the results obtained, the similarities and differences between public and private networks. Different aspects have been taken into account, such as; the level of fear in the contents, the topic used in the information and the time -past or future- referred to news and headlines. The main conclusion is that we can not set a clear distinction between the public and commercial channels.
\end{abstract}

Keywords: fear, news programme, television

\section{Referencia normalizada}

OSÉS GORRAIZ, Jesús María y BERMEJO AYESTRÁN, Aritz (2013): "El miedo en los informativos de televisión". Estudios sobre el Mensaje Periodístico. Vol. 19, Núm. 2 (julio-diciembre), págs.: 813-824. Madrid, Servicio de Publicaciones de la Universidad Complutense.

Sumario: 1. Introducción. 2. Hipótesis y objetivos. 3. Metodología. 4. Resultados; 4.1. Nivel de miedo; 4.2. Área temática; 4.3. Malas noticias o malos anuncios. 5. Conclusiones. 6. Referencias

\section{Introducción}

"Es el tiempo del miedo" constató Eduardo Galeano (La Jornada, 4/07/2006), en un artículo de prensa. En España eran todavía tiempos de bonanza y de euforia. Había seguridad en el presente y confianza en el futuro. Sin embargo, la crisis económica, política y moral desatada en los últimos años ha socavado los cimientos sobre los que se asentaba aquel optimismo y el desánimo se ha instalado en buena parte de los españoles.

La visión de nosotros mismos y del entorno ha cambiado. Y a ello han contribuido los medios de comunicación. Sin pretender caer en las teorías críticas, que conciben 
a los destinatarios como "una audiencia indefensa manipulada por los medios", y aun estando convencidos de "la capacidad de las personas para modificar el significado de los mensajes que reciben" (Castells, 2009: 178-179), no puede negarse su influencia en la ciudadanía.

Se podría decir que los medios de comunicación, con la selección temática y la presentación de los contenidos, contribuyen a generar expectativas -positivas o negativas- en la audiencia, lo que Gil Calvo (2003: 71) denomina climas de opinión. Estos se definirían como las "presunciones acerca del estado actual del sistema y sospechas sobre cuál será su posible tendencia futura", y fluctuarían entre el pánico y la euforia.

Ahora bien, ¿cómo contribuyen los medios a propagar el clima de miedo, esto es, la presunción de que las cosas están mal y la sospecha de que las cosas pueden empeorar? Gil Calvo concluye que lo hacen "tanto con malas noticias sobre lo que ya ha sucedido como con malos anuncios de posibles sorpresas escalofriantes que podrían estar a punto de ocurrir" (Ibídem: 164).

Tomando como referencia tal enunciado, en este trabajo nos proponemos averiguar si los medios (concretamente, las cadenas de televisión públicas y privadas) contribuyen en la misma medida y en la misma forma a la generación de la alarma social.

Se han comparado los informativos de las principales cadenas de televisión españolas (TVE 1 , Telecinco y Antena 3$)^{1}$ al objeto de comprobar si es posible establecer alguna diferencia entre la cadena pública (TVE 1), que es financiada mediante los presupuestos públicos, y las televisiones comerciales (Telecinco y Antena 3), que dependen de los ingresos publicitarios.

\section{Hipótesis y objetivos}

Partimos de una doble premisa. Por un lado, que las televisiones privadas apelan más a la emoción -"uno de los recursos fundamentales que permite a estos medios atraer y mantener la atención del espectador" (Conde, 1999: 59)- para, así, alcanzar mayores índices de audiencia y captar más anunciantes. Dado que una de las emociones básicas es el miedo, creemos que los canales comerciales infunden más miedo que el ente público.

Por otro lado, consideramos que las televisiones comerciales se apoyan prioritariamente en los sucesos y en los temas sociales para generar miedo -ámbitos que apelan más directamente a las emociones- y prestan una menor atención a la política y a la economía, áreas temáticas que encontrarían una mayor presencia en TVE 1, dado su carácter de "servicio esencial para la vida democrática" (RTVE, 2008: 1).

Para verificar estas hipótesis, nos hemos marcado un doble objetivo:

a) Cuantificar el nivel de miedo que infunden los informativos de cada cadena. Se establecerá de acuerdo con la duración de los contenidos.

1 En marzo de 2012, uno de los meses en los que se llevó a cabo el estudio, las audiencias medias de los informativos de las principales cadenas españolas fueron las siguientes: TVE encabezó la clasificación con un 17,2\% de cuota de pantalla, seguida de Telecinco (12,9\%), Antena 3 (12,4\%), Cuatro (6,4\%) y La Sexta (6,0\%) (Barlovento Comunicación, 2012: 13). 
b) Precisar las secciones en las que prioritariamente generan miedo, también de acuerdo con la duración de los contenidos. Se han fijado cuatro áreas temáticas: política, economía, sociedad y sucesos.

Además, a partir de la definición aportada por Gil Calvo, se determinará si el miedo procede mayormente de las malas noticias o de los malos anuncios.

Finalmente, se compararán los resultados obtenidos en cada canal al objeto de comprobar si existen similitudes significativas entre las cadenas comerciales y, en caso de ser así, verificar si se hallan diferencias sustanciales entre estas y el ente público. Para ello se extraerán los porcentajes, ya que la duración y el número de noticias varía de unos informativos a otros.

\section{Metodología ${ }^{2}$}

Se han analizado veintiún informativos, siete por cadena, utilizando el método de la semana compuesta para evitar así el excesivo protagonismo que un tema puede acaparar a lo largo de una semana. Los días seleccionados son el 13 (lunes), 21 (martes) y 29 (miércoles) de febrero; 8 (jueves), 16 (viernes) y 24 (sábado) de marzo; y 1 de abril (domingo), todos ellos correspondientes al año 2012.

De entre las dos ediciones principales (mediodía y noche), hemos optado por la de sobremesa que, si bien presenta unos índices de audiencia algo más bajos, metodológicamente ofrece más posibilidades ${ }^{3}$.

La unidad básica de análisis es el ítem informativo, que está integrado por diferentes elementos (por ejemplo, una presentación en estudio, una conexión en directo y un vídeo editado o una declaración). Cada ítem se refiere a un aspecto o motivo informativo, de manera que los diferentes contenidos incluidos en un bloque de breves se han considerado como ítems o unidades diferentes.

Se han estudiado por separado el sumario inicial y los avances internos (a los que denominamos titulares) y el resto de ítems (a los que llamamos noticias) para determinar hasta qué punto se destacan en los noticieros las informaciones que infunden miedo. No se han incluido las informaciones deportivas ni la predicción meteorológica, que en algunas cadenas se emiten en programas diferenciados.

Con respecto a la primera de las hipótesis (el nivel de miedo que infunden los canales), los contenidos se han clasificado en seis categorías: tres niveles básicos, que derivan del concepto propuesto por Gil Calvo, y otros tres que funcionan como gradientes.

2 Agradecemos a la periodista Cristina Aznar Munárriz el apoyo prestado en la delimitación de los aspectos metodológicos.

3 En marzo de 2012, los informativos de las tres cadenas seleccionadas sumaron de media 6.006.500 espectadores al mediodía, frente a los 6.385.500 de la noche (Barlovento Comunicación, 2012: 13). Sin embargo, los noticieros de sobremesa presentan formalmente una mayor similitud, en el sentido de que todos incluyen un bloque de titulares compacto, mientras que en el informativo nocturno de Telecinco el sumario es mínimo, lo que impide comparar sus resultados con los del resto de las cadenas. 
Las categorías básicas son la negativa (malas noticias y malos anuncios), la positiva (buenas noticias y buenos anuncios) y la neutra (ítems que no afectan a la visión que tenemos del mundo; por ejemplo, la visita de una celebridad a España o el estreno de una película). A ellas se suman la negativa compensada (malas noticias y anuncios con un atenuante o una reparación), la positiva matizada (buenas noticias y anuncios con algún aspecto que ensombrece la información) y la valorativa (ítems en los que se plasman las diferentes visiones de un asunto, más o menos, al mismo nivel; por ejemplo, las valoraciones políticas sobre un tema controvertido).

En el caso de las informaciones negativas y positivas, se ha especificado si se trata de malas o buenas noticias (sucesos ocurridos) o de malos o buenos anuncios (hechos supuestamente por acontecer).

En relación con la segunda hipótesis (las áreas temáticas en las que prioritariamente se genera miedo) se han establecido cuatro categorías a partir de la clasificación utilizada por Soengas ${ }^{4}$ : política, economía, sociedad y sucesos.

Aunque el trabajo de campo se llevó a cabo de acuerdo a una doble clasificación ${ }^{5}$, en este artículo se exponen los datos obtenidos en relación al enfoque o presentación de los contenidos. De esta manera, se entiende por política aquellas informaciones protagonizadas por las formaciones políticas y las instituciones del Estado; por economía, los ítems relacionados con los mercados, los precios, la empresa y el empleo; por sucesos, las catástrofes, los accidentes y, en un sentido amplio, las informaciones relacionadas con la seguridad y las emergencias; y por sociedad, el resto: arte y cultura, medio ambiente, salud, consumo, estilos de vida, religión, ciencia y tecnología, y temas sociales.

\section{Resultados}

Se han analizado un total de 14 horas 2 minutos y 23 segundos de informativo, de los que 5 horas 37 minutos y 15 segundos corresponden a TVE 1, la cadena con los noticieros más largos; 4 horas 18 minutos y 6 segundos, a Telecinco; y 4 horas 7 minutos y 2 segundos, a Antena 3.

${ }^{4}$ Soengas (2007: 328). agrupa los contenidos en seis secciones “consideradas clásicas": política, economía, sociedad, sucesos, nacional e internacional. Las otras dos categorías, nacional e internacional, se han empleado en el trabajo de campo para clasificar los ítems por área geográfica.

${ }^{5}$ Se diferenció entre la temática y la presentación de los contenidos, distinguiendo entre un concepto amplio de la política (cualquier actividad relacionada con los asuntos públicos) y otro stricto sensu (las informaciones protagonizadas por las formaciones políticas y las instituciones del Estado). Ello nos permitió diferenciar entre aquellas informaciones que son políticas (temática) de lo que popularmente se entiende por política (presentación). Por ejemplo, los bombardeos sobre la ciudad de Homs, en Siria, son una cuestión política, pero su presentación se asemeja más a la de un suceso, ya que rara vez se hace referencia al conflicto de fondo y solo suelen citarse los civiles fallecidos en el día, como si de un asesinato colectivo se tratara. Así, el concepto amplio de política se subdividió en política stricto sensu, economía, sociedad y sucesos para determinar la presentación de los temas políticos. En las tres últimas categorías (economía, sociedad y sucesos) los resultados se sumaron a los obtenidos en la clasificación temática. 
Con respecto a TVE 1, se han estudiado 54 titulares, con una duración de 28 minutos y 32 segundos, y 233 noticias, que suman 5 horas 4 minutos y 43 segundos. En Telecinco, se han examinado 61 titulares, con un tiempo de 28 minutos, y 227 noticias, que computan 3 horas 46 minutos y 25 segundos. Y en Antena 3, se han analizado 63 titulares, con una duración de 25 minutos y 2 segundos, y 192 titulares, que suman 3 horas 37 minutos y 21 segundos. El resto del tiempo -4 minutos en TVE 1, 3 minutos y 41 segundos en Telecinco, y 4 minutos y 39 segundos en Antena 3- corresponden a caretas de entrada y de salida, ráfagas, etc.

Se observa que los informativos del ente público tienen los titulares más largos, con una media de 31 segundos, seguidos por los de Telecinco (27 segundos) y Antena 3 ( 23 segundos). TVE 1 es también la cadena con las noticias más extensas, con una duración media de 1 minuto y 18 segundos, por delante de Antena 3 ( 1 minuto y 7 segundos) y Telecinco (59 segundos).

Se aprecia, por otro lado, una modesta coincidencia en los temas seleccionados por las cadenas. Si comparamos los contenidos incluidos por los tres canales, la concurrencia en ningún caso alcanza el $35 \%$. La media es del $23,59 \%$ en los titulares (del $20 \%$ de lunes a viernes y del $34,88 \%$ el fin de semana) y del $32,21 \%$ en las noticias (del 33,26\% de lunes a viernes y del 29,09\% el fin de semana).

\subsection{Nivel de miedo}

En todas las cadenas el enfoque mayoritario es el negativo (malas noticias o malos anuncios), tanto en titulares como en noticias, como se observa en el cuadro I. Esta categoría aglutina grosso modo uno de cada dos ítems.

En el caso de los titulares, los informativos con mayor porcentaje de ítems negativos son los de Antena 3 (61,72\%), seguidos de cerca por los de Telecinco $(59,34 \%)$ y a distancia por los de TVE $1(48,31 \%)$; mientras que en el caso de las noticias, los informativos que incluyen proporcionalmente más ítems negativos son los de Telecinco $(58,93 \%)$, seguidos por los de TVE $1(55,31 \%)$ y los de Antena $3(49,06 \%)$.

En todos los casos se aprecia una mayor preferencia por los temas negativos de lunes a viernes. Destaca Telecinco, sobre todo en titulares, donde los niveles descienden de manera notable en fin de semana (41 puntos en titulares y 26 en noticias).

\begin{tabular}{|c|c|c|c|c|c|c|c|}
\hline \multicolumn{8}{|c|}{ TITULARES } \\
\hline & & Negativo & $\begin{array}{c}\text { Negativo } \\
\text { compensado }\end{array}$ & Valorativo & $\begin{array}{c}\text { Positivo } \\
\text { matizado }\end{array}$ & Positivo & Neutro \\
\hline \multirow[t]{3}{*}{ TVE 1} & & $48,31 \%$ & $4,20 \%$ & $23,77 \%$ & $10,40 \%$ & $4,09 \%$ & $9,23 \%$ \\
\hline & $\mathrm{I}-\mathrm{v}$ & $49,68 \%$ & $5,66 \%$ & $25,86 \%$ & $7,63 \%$ & $5,50 \%$ & $5,66 \%$ \\
\hline & s-d & $44,32 \%$ & $0 \%$ & $17,73 \%$ & $18,41 \%$ & $0 \%$ & $19,54 \%$ \\
\hline \multirow[t]{3}{*}{ T5 } & & $59,34 \%$ & $8,09 \%$ & $3,27 \%$ & $2,98 \%$ & $1,49 \%$ & $24,82 \%$ \\
\hline & $\mathrm{I}-\mathrm{v}$ & $72,61 \%$ & $5,44 \%$ & $1,76 \%$ & $0 \%$ & $2,19 \%$ & $18,00 \%$ \\
\hline & s-d & $31,42 \%$ & $13,68 \%$ & $6,47 \%$ & $9,24 \%$ & $0 \%$ & $39,19 \%$ \\
\hline \multirow[t]{3}{*}{ A3 } & & $61,72 \%$ & $1,80 \%$ & $19,04 \%$ & $1,53 \%$ & $3,33 \%$ & $12,58 \%$ \\
\hline & $\mathrm{I}-\mathrm{v}$ & $62,85 \%$ & $2,19 \%$ & $19,87 \%$ & $1,87 \%$ & $4,06 \%$ & $9,16 \%$ \\
\hline & s-d & $56,50 \%$ & $0 \%$ & $15,24 \%$ & $0 \%$ & $0 \%$ & $28,25 \%$ \\
\hline \multicolumn{8}{|c|}{ NOTICIAS } \\
\hline \multirow[t]{3}{*}{ TVE 1} & & $55,31 \%$ & $5,60 \%$ & $10,86 \%$ & $8,38 \%$ & $3,65 \%$ & $16,19 \%$ \\
\hline & I-v & $58,54 \%$ & $6,70 \%$ & $11,87 \%$ & $5,65 \%$ & $4,77 \%$ & $12,47 \%$ \\
\hline & $s-d$ & $44,80 \%$ & $2,03 \%$ & $7,58 \%$ & $17,28 \%$ & $0 \%$ & $28,31 \%$ \\
\hline \multirow[t]{3}{*}{ T5 } & & $58,93 \%$ & $9,53 \%$ & $5,54 \%$ & $2,92 \%$ & $1,57 \%$ & $21,50 \%$ \\
\hline & $\mathrm{I}-\mathrm{v}$ & $66,23 \%$ & $10,66 \%$ & $6,16 \%$ & $0,98 \%$ & $2,19 \%$ & $13,77 \%$ \\
\hline & $s-d$ & $40,69 \%$ & $6,72 \%$ & $3,99 \%$ & $7,78 \%$ & $0 \%$ & $40,82 \%$ \\
\hline \multirow[t]{3}{*}{ A3 } & & $49,06 \%$ & $9,45 \%$ & $6,69 \%$ & $3,92 \%$ & $6,27 \%$ & $24,60 \%$ \\
\hline & $\mathrm{I}-\mathrm{v}$ & $53,12 \%$ & $12,17 \%$ & $8,68 \%$ & $3,71 \%$ & $8,53 \%$ & $13,78 \%$ \\
\hline & $s-d$ & $38,82 \%$ & $2,62 \%$ & $1,67 \%$ & $4,45 \%$ & $0,57 \%$ & $51,86 \%$ \\
\hline
\end{tabular}


Se observa, por otro lado, que los ítems positivos (buenas noticias o buenos anuncios) son escasos. En los titulares, TVE 1 es la cadena con más temas positivos, con el $4,09 \%$ del total, por encima de Antena 3 (el 3,33\%) y Telecinco (el 1,49\%). En las noticias, en cambio, Antena 3 encabeza el ranking, con un $6,27 \%$, frente al 3,65\% de TVE 1 y al 1,57\% de Telecinco.

Llama la atención que prácticamente todos los ítems positivos han sido emitidos de lunes a viernes. Únicamente existe una noticia positiva en fin de semana. Fue emitida por Antena 3 el 1 de abril (domingo) y, curiosamente, fue matizada a continuación por otra noticia negativa que guardaba relación con la anterior.

Se aprecia, por tanto, que el descenso de ítems negativos el sábado y el domingo no implica un incremento de los positivos. La categoría que acapara estas caídas es, mayormente, la neutra, hasta el punto de aglutinar más de la mitad de las noticias emitidas el fin de semana en Antena 3 (el 51,86\%).

Este incremento es menor en TVE $1 \mathrm{y}$, con ello, el ente público es la cadena que proporcionalmente más noticias negativas emite el sábado y el domingo: 44,80\% frente al $40,69 \%$ de Telecinco y al $38,82 \%$ de Antena 3 .

El enfoque neutro tiene un mayor peso en los informativos de las cadenas privadas. En cualquier caso, en todas -incluida la pública- es el segundo en importancia en noticias: $24,60 \%$ en Antena 3, 21,50\% en Telecinco y $16,19 \%$ en TVE 1.

Lo mismo ocurre en titulares con Telecinco (24,82\%), pero no con TVE $1(9,23 \%)$ y Antena $3(12,58 \%)$, cadenas en las que el enfoque valorativo ocupa la segunda posición, por detrás del negativo, con un $23,77 \%$ y $19,04 \%$, respectivamente. El neutro ocupa el tercer puesto en Antena 3 y el cuarto en el ente público, por detrás del positivo matizado $(10,40 \%)$.

\section{2. Área temática}

Comparativamente, las áreas de política y economía tienen mayor peso en la cadena pública y las secciones de sociedad y sucesos, en las televisiones privadas.

Como se precisa en el cuadro II, el área de política es la principal en TVE 1 ( $43,05 \%$ de titulares y $37,03 \%$ de noticias), seguida por la de sucesos en titulares $(23,36 \%)$ y la de sociedad en noticias $(30,25 \%)$. En Telecinco la sección que más ítems agrupa es sociedad ( $48,87 \%$ de titulares y $42,33 \%$ de noticias) y, después, sucesos (33,09\% de titulares y $36,17 \%$ de noticias). Y en Antena 3 destacan, en titulares, las secciones de sucesos $(37,15 \%)$ y de política $(29,43 \%)$-esta última ligeramente superior a la de sociedad $(27,16 \%)$ - y, en noticias, las de sociedad $(35,89 \%)$ y suce$\operatorname{sos}(32,27 \%)$.

Economía es la categoría menos frecuente en todas. No obstante, dentro de su modesta presencia, se aprecia que tiene un mayor peso en la cadena pública, cuyos resultados prácticamente doblan la media de las televisiones privadas.

A pesar de estas diferencias en los datos globales, observamos una notable coincidencia en los ítems negativos. Por lo general, el área de sucesos es la que más malas noticias y malos anuncios agrupa: el 60,19\% de los titulares en Antena 3, el 53,56\% en Telecinco y el 44,50\% en TVE 1, así como el 62,49\% de las noticias en Antena 3 y el $56,22 \%$ en Telecinco. 
Existe una única excepción, la correspondiente a las noticias del ente público, donde la mayor parte de los ítems negativos pertenecen al área de política $(37,40 \%)$, aunque seguida de cerca por la de sucesos $(35,58 \%)$.

El caso es que la sección de política es mayoritaria en esta cadena de lunes a viernes $(39,72 \%$ de titulares y $44,13 \%$ de noticias), mientras que la de sucesos aumenta de forma considerable en fin de semana $(88,72 \%$ de titulares y $73,97 \%$ de noticias), hasta el

\begin{tabular}{|c|c|c|c|c|c|c|c|c|}
\hline \multicolumn{5}{|c|}{ TITULARES } & \multicolumn{4}{|c|}{ NOTICIAS } \\
\hline & Pol. & Eco. & Soc. & Suc. & Pol. & Eco. & Soc. & Suc. \\
\hline \multicolumn{9}{|c|}{ Datos globales } \\
\hline TVE 1 & $43,05 \%$ & $12,32 \%$ & $21,26 \%$ & $23,36 \%$ & $37,03 \%$ & $12,05 \%$ & $30,25 \%$ & $20,66 \%$ \\
\hline I-v & $50,55 \%$ & $12,73 \%$ & $18,87 \%$ & $17,85 \%$ & $42,95 \%$ & $14,60 \%$ & $25,96 \%$ & $16,49 \%$ \\
\hline s-d & $21,36 \%$ & $11,14 \%$ & $28,18 \%$ & $39,32 \%$ & $17,72 \%$ & $3,73 \%$ & $44,26 \%$ & $34,28 \%$ \\
\hline T5 & $11,67 \%$ & $6,37 \%$ & $48,87 \%$ & $33,09 \%$ & $17,10 \%$ & $4,40 \%$ & $42,33 \%$ & $36,17 \%$ \\
\hline $1-v$ & $14,13 \%$ & $6,06 \%$ & $37,93 \%$ & $41,88 \%$ & $18,20 \%$ & $4,45 \%$ & $35,06 \%$ & $42,28 \%$ \\
\hline s-d & $6,47 \%$ & $7,02 \%$ & $71,90 \%$ & $14,60 \%$ & $14,34 \%$ & $4,27 \%$ & $60,47 \%$ & $20,91 \%$ \\
\hline A3 & $29,43 \%$ & $6,26 \%$ & $27,16 \%$ & $37,15 \%$ & $24,21 \%$ & $7,62 \%$ & $35,89 \%$ & $32,27 \%$ \\
\hline $1-v$ & $30,90 \%$ & $7,62 \%$ & $23,52 \%$ & $37,96 \%$ & $28,49 \%$ & $9,97 \%$ & $26,54 \%$ & $35,00 \%$ \\
\hline s-d & $22,68 \%$ & $0 \%$ & $43,87 \%$ & $33,46 \%$ & $13,44 \%$ & $1,70 \%$ & $59,45 \%$ & $25,40 \%$ \\
\hline \multicolumn{9}{|c|}{ Enfoque negativo } \\
\hline TVE 1 & $30,35 \%$ & $6,89 \%$ & $18,26 \%$ & $44,50 \%$ & $37,40 \%$ & $9,95 \%$ & $17,08 \%$ & $35,58 \%$ \\
\hline $1-v$ & $39,72 \%$ & $9,02 \%$ & $20,41 \%$ & $30,85 \%$ & $44,13 \%$ & $12,28 \%$ & $17,02 \%$ & $26,57 \%$ \\
\hline s-d & $0 \%$ & $0 \%$ & $11,28 \%$ & $88,72 \%$ & $8,69 \%$ & $0 \%$ & $17,33 \%$ & $73,97 \%$ \\
\hline T5 & $12,64 \%$ & $6,92 \%$ & $26,88 \%$ & $53,56 \%$ & $15,63 \%$ & $3,51 \%$ & $24,64 \%$ & $56,22 \%$ \\
\hline $1-v$ & $15,24 \%$ & $8,34 \%$ & $18,74 \%$ & $57,68 \%$ & $17,07 \%$ & $3,24 \%$ & $20,17 \%$ & $59,52 \%$ \\
\hline s-d & $0 \%$ & $0 \%$ & $66,47 \%$ & $33,53 \%$ & $9,75 \%$ & $4,62 \%$ & $42,85 \%$ & $42,78 \%$ \\
\hline A3 & $21,90 \%$ & $3,45 \%$ & $14,46 \%$ & $60,19 \%$ & $20,07 \%$ & $8,02 \%$ & $9,42 \%$ & $62,49 \%$ \\
\hline $1-v$ & $23,61 \%$ & $4,13 \%$ & $11,87 \%$ & $60,39 \%$ & $19,84 \%$ & $10,34 \%$ & $8,19 \%$ & $61,63 \%$ \\
\hline$s-d$ & $13,16 \%$ & $0 \%$ & $27,63 \%$ & $59,21 \%$ & $20,86 \%$ & $0 \%$ & $13,70 \%$ & $65,44 \%$ \\
\hline \multicolumn{9}{|c|}{ Enfoque positivo } \\
\hline TVE 1 & $47,14 \%$ & $20 \%$ & $32,86 \%$ & $0 \%$ & $27,25 \%$ & $19,76 \%$ & $52,99 \%$ & $0 \%$ \\
\hline $1-v$ & $47,14 \%$ & $20 \%$ & $32,86 \%$ & $0 \%$ & $27,25 \%$ & $19,76 \%$ & $52,99 \%$ & $0 \%$ \\
\hline s-d & $0 \%$ & $0 \%$ & $0 \%$ & $0 \%$ & $0 \%$ & $0 \%$ & $0 \%$ & $0 \%$ \\
\hline T5 & $0 \%$ & $0 \%$ & $100 \%$ & $0 \%$ & $10,33 \%$ & $5,16 \%$ & $84,51 \%$ & $0 \%$ \\
\hline $1-v$ & $0 \%$ & $0 \%$ & $100 \%$ & $0 \%$ & $10,33 \%$ & $5,16 \%$ & $84,51 \%$ & $0 \%$ \\
\hline s-d & $0 \%$ & $0 \%$ & $0 \%$ & $0 \%$ & $0 \%$ & $0 \%$ & $0 \%$ & $0 \%$ \\
\hline A3 & $0 \%$ & $0 \%$ & $100 \%$ & $0 \%$ & $21,03 \%$ & $6,11 \%$ & $69,80 \%$ & $3,06 \%$ \\
\hline $1-v$ & $0 \%$ & $0 \%$ & $100 \%$ & $0 \%$ & $21,58 \%$ & $6,27 \%$ & $69,01 \%$ & $3,14 \%$ \\
\hline s-d & $0 \%$ & $0 \%$ & $0 \%$ & $0 \%$ & $0 \%$ & $0 \%$ & $100 \%$ & $0 \%$ \\
\hline
\end{tabular}
Elaboración propia

punto de superar los porcentajes de las cadenas privadas (Telecinco: $33,53 \%$ de titulares y $42,78 \%$ de noticias; y Antena 3: 59,21\% de titulares y $65,44 \%$ de noticias).

En Telecinco ocurre lo contrario. La categoría de sucesos, que es la principal entre semana (57,68\% de titulares y 59,52\% de noticias), registra un descenso significativo el sábado y el domingo en favor del área de sociedad $(66,47 \%$ de titulares y $42,85 \%$ de noticias), de media, la segunda en importancia. Prácticamente uno de cada cuatro ítems negativos pertenecen a esta sección.

En Antena 3, el área de sucesos es mayoritaria, tanto de lunes a viernes como en fin de semana, y la segunda es la de política $(21,90 \%$ de titulares y $20,07 \%$ de noticias), notablemente por encima de la de sociedad ( $14,46 \%$ de titulares y $9,42 \%$ de noticias). Se diferencia, así, de Telecinco, donde la sección de política registra porcentajes más modestos ( $12,64 \%$ de titulares y $15,63 \%$ de noticias).

Los valores del área de economía son los más bajos, algo lógico, por otro lado, debido a que las informaciones de carácter económico son las menos frecuentes. En todas las clasificaciones realizadas los porcentajes rara vez superan el $10 \%$.

Y ¿qué ocurre con los ítems positivos? Aquí también existe una amplia coincidencia entre las cadenas. La mayoría de las buenas noticias y de los buenos anuncios pertenecen, por lo general, al área de sociedad: en titulares, el 100\% de los ítems en las cadenas privadas y, en noticias, el $84,51 \%$ de las informaciones en Telecinco, el $69,80 \%$ en Antena 3 y el 52,99\% en TVE 1. 
Como ocurría con los ítems negativos, existe una excepción, en este caso, con los titulares de la cadena pública, donde el área de política encabeza el ranking $(47,14 \%)$, por delante de las de sociedad (32,86\%) y economía (20\%). Esta situación no se debe a una mayor presencia de temas políticos en los titulares positivos, ya que en las fechas analizadas, se emitieron tres, uno por área. Simplemente que el de política tuvo mayor duración.

Por otra parte, se constata que prácticamente no existen sucesos positivos, circunstancia normal, debido a que estos hacen referencia básicamente a accidentes, catástrofes y actos delictivos. Casualmente se ha encontrado uno en Antena 3, un tema político con la presentación de suceso: la captura del último cabecilla de Sendero Luminoso, con la que se daba por extinguida la organización.

Esta circunstancia se evidencia en el cuadro III, donde se expone el enfoque de los ítems, clasificados en función de las áreas temáticas. En todas las cadenas, más del $90 \%$ de los titulares y noticias de la sección de sucesos tienen un enfoque negativo.

En el área de política sobresalen los enfoques negativo y valorativo, este último especialmente en titulares, donde llega a ser el mayoritario en TVE 1 $(38,53 \%)$. En el resto de los casos, el enfoque principal es el negativo, seguido del valorativo $y$, a más distancia, por el negativo compensado y positivo matizado. Hay pocas informaciones positivas y neutras.

En la sección de economía sucede algo parecido. En los titulares destacan los enfoques negativo (principal en Telecinco) y valorativo (principal en TVE $1 \mathrm{y}$ Antena 3). Sin embargo, esta posición intermedia en titulares se decanta, fundamentalmente, en favor del enfoque negativo $o$ negativo compensado en las noticias, las cate-

\begin{tabular}{|c|c|c|c|c|c|c|}
\hline \multicolumn{7}{|c|}{ TITULARES } \\
\hline & Negativo & $\begin{array}{c}\text { Negativo } \\
\text { compensado }\end{array}$ & Valorativo & $\begin{array}{l}\text { Positivo } \\
\text { matizado }\end{array}$ & Positivo & Neutro \\
\hline \multicolumn{7}{|l|}{ Politica } \\
\hline TVE 1 & $34,06 \%$ & $9,77 \%$ & $38,53 \%$ & $13,16 \%$ & $4,47 \%$ & $0 \%$ \\
\hline TS & $64,29 \%$ & $17,86 \%$ & $17,86 \%$ & $0 \%$ & $0 \%$ & $0 \%$ \\
\hline$A 3$ & $45,93 \%$ & $6,11 \%$ & $42,75 \%$ & $5,20 \%$ & $0 \%$ & $0 \%$ \\
\hline \multicolumn{7}{|l|}{ Economía } \\
\hline TVE 1 & $27,01 \%$ & $0 \%$ & $43,13 \%$ & $23,22 \%$ & $6,64 \%$ & $0 \%$ \\
\hline TS & $64,49 \%$ & $35,51 \%$ & $0 \%$ & $0 \%$ & $0 \%$ & $0 \%$ \\
\hline A3 & $34,04 \%$ & $0 \%$ & $65,96 \%$ & $0 \%$ & $0 \%$ & $0 \%$ \\
\hline \multicolumn{7}{|l|}{ Sociedad } \\
\hline TVE 1 & $41,48 \%$ & $0 \%$ & $8,79 \%$ & $0 \%$ & $6,32 \%$ & $43,41 \%$ \\
\hline T5 & $32,64 \%$ & $7,67 \%$ & $2,44 \%$ & $3,41 \%$ & $3,05 \%$ & $50,79 \%$ \\
\hline A3 & $32,84 \%$ & $0 \%$ & $8,58 \%$ & $0 \%$ & $12,25 \%$ & $46,32 \%$ \\
\hline \multicolumn{7}{|l|}{ Sucesos } \\
\hline TVE 1 & $92 \%$ & $0 \%$ & $0 \%$ & $8 \%$ & $0 \%$ & $0 \%$ \\
\hline T5 & $96,04 \%$ & $0 \%$ & $0 \%$ & $3,96 \%$ & $0 \%$ & $0 \%$ \\
\hline A3 & $100 \%$ & $0 \%$ & $0 \%$ & $0 \%$ & $0 \%$ & $0 \%$ \\
\hline \multicolumn{7}{|c|}{ NOTICIAS } \\
\hline & Negativo & $\begin{array}{c}\text { Negativo } \\
\text { compensado }\end{array}$ & Valorativo & $\begin{array}{l}\text { Positivo } \\
\text { matizado }\end{array}$ & Positivo & Neutro \\
\hline \multicolumn{7}{|l|}{ Política } \\
\hline TVE 1 & $55,86 \%$ & $10,19 \%$ & $16,69 \%$ & $13,28 \%$ & $2,69 \%$ & $1,30 \%$ \\
\hline T5 & $53,85 \%$ & $11,97 \%$ & $21,52 \%$ & $4,05 \%$ & $0,95 \%$ & $7,66 \%$ \\
\hline A3 & $40,66 \%$ & $16,18 \%$ & $21,25 \%$ & $13,33 \%$ & $5,45 \%$ & $3,13 \%$ \\
\hline \multicolumn{7}{|l|}{ Economía } \\
\hline TVE 1 & $45,67 \%$ & $15,16 \%$ & $17,29 \%$ & $15,89 \%$ & $5,99 \%$ & $0 \%$ \\
\hline T5 & $46,99 \%$ & $43,14 \%$ & $0 \%$ & $8,03 \%$ & $1,84 \%$ & $0 \%$ \\
\hline A3 & $51,61 \%$ & $34,31 \%$ & $0 \%$ & $9,05 \%$ & $5,03 \%$ & $0 \%$ \\
\hline \multicolumn{7}{|l|}{ Sociedad } \\
\hline TVE 1 & $31,22 \%$ & $0 \%$ & $8,59 \%$ & $1,86 \%$ & $6,40 \%$ & $51,93 \%$ \\
\hline T5 & $34,31 \%$ & $8,38 \%$ & $4,40 \%$ & $2,07 \%$ & $3,13 \%$ & $47,70 \%$ \\
\hline A3 & $12,88 \%$ & $4,17 \%$ & $4,32 \%$ & $0 \%$ & $12,20 \%$ & $66,43 \%$ \\
\hline \multicolumn{7}{|l|}{ Sucesos } \\
\hline TVE 1 & $95,24 \%$ & $0 \%$ & $0 \%$ & $4,76 \%$ & $0 \%$ & $0 \%$ \\
\hline T5 & $91,60 \%$ & $5,64 \%$ & $0 \%$ & $2,77 \%$ & $0 \%$ & $0 \%$ \\
\hline A3 & $94,99 \%$ & $4,42 \%$ & $0 \%$ & $0 \%$ & $0,59 \%$ & $0 \%$ \\
\hline
\end{tabular}

Elaboración propia 
gorías mayoritarias. Apenas hay informaciones económicas positivas y no hay ninguna neutra.

Estos datos confirman que los bajos índices de noticias económicas negativas en los informativos se deben más a la modesta presencia de estas informaciones en los noticieros que a su enfoque.

Por último, en los ítems del área de sociedad sobresalen el enfoque neutro (el principal en todas las categorías) y el negativo. A pesar de ser la sección que más informaciones positivas agrupa, el cuadro III evidencia que este enfoque no es significativo en el global de las informaciones de carácter social.

\subsection{Malas noticias o malos anuncios}

La mayoría de los ítems analizados hacen referencia a hechos acaecidos: el 68,46\% de los titulares en TVE 1, el 89,23\% en Telecinco y el 83,22\% en Antena 3, así como el $82,81 \%$ de las noticias en TVE 1 , el $91,52 \%$ en Telecinco y el 84,19\% en Antena 3.

De acuerdo con estos datos, es lógico que la mayoría de las informaciones negativas se refieran a acontecimientos pasados. Los valores en las televisiones privadas superan el $90 \%$ y en el ente público quedan por encima del $80 \%$.

Esta tendencia es todavía más evidente en los ítems de carácter positivo. En los titulares, todos los contenidos versan sobre hechos ocurridos, de manera que no hay pronósticos positivos, y en las noticias, únicamente existen dos, ambos emitidos por la cadena pública.

\section{Conclusiones}

Al analizar los datos se observa que no puede dibujarse una clara línea de separación entre la televisión pública y las cade-

\begin{tabular}{|c|c|c|c|c|}
\hline & \multicolumn{2}{|c|}{ TITULARES } & \multicolumn{2}{|c|}{ NOTICIAS } \\
\hline & Noticia & Anuncio & Noticia & Anuncio \\
\hline \multicolumn{5}{|l|}{ Datos globales } \\
\hline \multirow[t]{3}{*}{ TVE 1} & $68,46 \%$ & $31,54 \%$ & $82,81 \%$ & $17,18 \%$ \\
\hline & $64,70 \%$ & $35,30 \%$ & $81,06 \%$ & $18,94 \%$ \\
\hline & $79,32 \%$ & $20,68 \%$ & $88,55 \%$ & $11,45 \%$ \\
\hline \multirow[t]{3}{*}{ T5 } & $89,23 \%$ & $10,77 \%$ & $91,52 \%$ & $8,48 \%$ \\
\hline & $92,71 \%$ & $7,29 \%$ & $91,76 \%$ & $8,23 \%$ \\
\hline & $81,88 \%$ & $18,11 \%$ & $90,91 \%$ & $9,09 \%$ \\
\hline \multirow[t]{3}{*}{ A3 } & $83,22 \%$ & $16,78 \%$ & $84,19 \%$ & $15,81 \%$ \\
\hline & $82,89 \%$ & $17,11 \%$ & $80,38 \%$ & $19,62 \%$ \\
\hline & $84,76 \%$ & $15,24 \%$ & $93,79 \%$ & $6,21 \%$ \\
\hline \multicolumn{5}{|c|}{ Enfoque negativo } \\
\hline \multirow[t]{3}{*}{ TVE 1} & $81,86 \%$ & $18,14 \%$ & $88,19 \%$ & $11,81 \%$ \\
\hline & $83,07 \%$ & $16,93 \%$ & $86,08 \%$ & $13,92 \%$ \\
\hline & $77,95 \%$ & $22,05 \%$ & $97,19 \%$ & $2,81 \%$ \\
\hline \multirow[t]{3}{*}{ T5 } & $97,89 \%$ & $2,11 \%$ & $94,69 \%$ & $5,31 \%$ \\
\hline & $97,46 \%$ & $2,54 \%$ & $95,83 \%$ & $4,17 \%$ \\
\hline & $100 \%$ & $0 \%$ & $90,06 \%$ & $9,94 \%$ \\
\hline \multirow[t]{3}{*}{ A3 } & $92,45 \%$ & $7,55 \%$ & $90,29 \%$ & $9,71 \%$ \\
\hline & $90,97 \%$ & $9,03 \%$ & $89,44 \%$ & $10,56 \%$ \\
\hline & $100 \%$ & $0 \%$ & $93,25 \%$ & $6,75 \%$ \\
\hline \multicolumn{5}{|c|}{ Enfoque positivo } \\
\hline \multirow[t]{3}{*}{ TVE 1} & $100 \%$ & $0 \%$ & $90,12 \%$ & $9,88 \%$ \\
\hline & $100 \%$ & $0 \%$ & $90,12 \%$ & $9,88 \%$ \\
\hline & $0 \%$ & $0 \%$ & $0 \%$ & $0 \%$ \\
\hline \multirow[t]{3}{*}{ T5 } & $100 \%$ & $0 \%$ & $100 \%$ & $0 \%$ \\
\hline & $100 \%$ & $0 \%$ & $100 \%$ & $0 \%$ \\
\hline & $0 \%$ & $0 \%$ & $0 \%$ & $0 \%$ \\
\hline \multirow[t]{3}{*}{ A3 } & $100 \%$ & $0 \%$ & $100 \%$ & $0 \%$ \\
\hline & $100 \%$ & $0 \%$ & $100 \%$ & $0 \%$ \\
\hline & $0 \%$ & $0 \%$ & $100 \%$ & $0 \%$ \\
\hline
\end{tabular}
nas privadas. Las tres coinciden en muchos aspectos y cuando difieren, las diferencias no tienen por qué responder a su carácter público o comercial. De hecho, hay parámetros en los que TVE 1 tiene mayor similitud con alguno de los canales privados que la que tienen ellos entre sí. Por tanto, quedan refutadas, en parte, las hipótesis que planteábamos al inicio de este trabajo.

La primera, la relativa al nivel de miedo, podría mantenerse para los titulares, donde TVE 1 es el canal con menos informaciones negativas (48,31\% frente al $61,72 \%$ de Antena 3 y al 59,34\% de Telecinco). No así para las noticias, donde el ente público presenta, con carácter general, una posición intermedia $(55,31 \%$ frente al $49,06 \%$ de 
Antena 3 y al 58,93\% de Telecinco) e, incluso, llega a superar a las cadenas comerciales el fin de semana $(44,80 \%$ frente al $38,82 \%$ de Antena 3 y al $40,69 \%$ de Telecinco).

La segunda, la que hace referencia a las áreas temáticas, también queda parcialmente desmentida. Atendiendo a los contenidos generales del informativo, sí que se aprecia una mayor presencia de temas políticos y económicos en TVE 1, y de contenidos sociales y de sucesos en las televisiones privadas. Ahora bien, reparando únicamente en los negativos, se observa que no siempre se cumple esta premisa. Un ejemplo: en TVE 1 la sección de sucesos experimenta un fuerte incremento el fin de semana hasta convertirse en la principal en la propagación del miedo, con valores que, incluso, superan a los de las cadenas comerciales, tanto en los titulares $(88,72 \%$ frente al 59,21\% de Antena 3 y al 33,53\% de Telecinco) como en las noticias (73,97\% frente al $65,44 \%$ de Antena 3 y al $42,78 \%$ de Telecinco). Asimismo, en Antena 3, siendo la sección de sucesos principal en los ítems negativos, la de política ocupa la segunda posición, por encima de la de sociedad (en titulares, $21,90 \%$ frente a $14,46 \% \mathrm{y}$, en noticias, $20,07 \%$ frente a 9,42\%); y en Telecinco, el valor del área económica $(6,92 \%)$ supera ligeramente a la de TVE $1(6,89 \%)$ en titulares.

Matizadas ambas hipótesis, veamos ahora de manera esquemática otras conclusiones que también se extraen de los resultados obtenidos:

1. Los informativos incluyen mayoritariamente contenidos negativos, sobre todo entre semana, ya que el fin de semana aumentan de forma considerable las informaciones neutras. Por otro lado, apenas existen informaciones positivas y casi todas son emitidas de lunes a viernes. Esto supone que el descenso registrado en los contenidos negativos el fin de semana no conlleva un aumento de los contenidos positivos, sino de los neutros.

2. Se podría pensar que esta coincidencia en el enfoque pudiera deberse a que las televisiones informan sobre unos mismos temas que, desde el punto de vista periodístico, son de interés general. Sin embargo, como se ha visto, la coincidencia temática no llega en ningún caso al 35\%. Por tanto, la presencia mayoritaria de los temas negativos en los informativos, con valores superiores al $35 \%$, no se debe tanto a este planteamiento como a la preferencia de los medios por las informaciones negativas. Se confirma, así, la máxima periodística, según la cual "las malas noticias son buenas noticias".

3. Los resultados vienen también a corroborar que el nivel de negatividad de las informaciones no depende del tiempo que se les asigna. Se podría creer que una noticia más extensa pudiera generar menos miedo, en el sentido de que tiene más tiempo para profundizar en la información y, con ello, para darla a entender en su contexto. Como se ha indicado, la carga de negatividad en TVE 1 es parecida a la del resto de las cadenas (en la categoría noticias), cuando sus informaciones son más extensas. Parece, pues, que el enfoque no depende tanto de la cantidad de información o datos que se ofrecen como de la selección temática y de su interpretación.

4. Las televisiones generan miedo, especialmente, a través de las áreas de política y de sucesos. Llama la atención que en un momento como el actual, en el que los 
temas económicos acaparan tantas portadas, la sección de economía presente unos valores tan bajos. Como se ha indicado, este hecho se debe a la escasa presencia de las informaciones económicas, más que a su enfoque, que en muchos casos es negativo. Pero, aun considerando la posibilidad de que el área de política absorba parte de esas informaciones, parece poco probable, con estos datos en mano, que la de economía sea la principal fuente de alarma social en las televisiones (la suma de los porcentajes de las áreas de política y economía es inferior a los de la sección de sucesos, salvo alguna excepción en TVE 1).

5. Por otro lado, la mayoría de los contenidos positivos corresponden al área de sociedad, una sección que también destaca por el carácter mayoritariamente neutro de sus informaciones.

6. Se aprecia una tendencia a finalizar los informativos con temas sociales de carácter neutro. Parece que los editores buscan para el final de sus noticieros temas más amables que suavizan la carga negativa acumulada a lo largo del informativo.

7. Las televisiones informan fundamentalmente sobre hechos ocurridos y, consecuentemente, propagan el miedo sobre todo a través de sucesos acontecidos. Entre los anuncios apenas hay pronósticos positivos.

Hay que tener en cuenta que estas conclusiones hacen referencia a un contexto concreto (una profunda crisis económica y un modelo de gestión despolitizado de la Corporación RTVE), por lo que sería recomendable realizar en el futuro estudios similares para confirmar si las principales tendencias observadas son permanentes o coyunturales. Por otro lado, hay que recordar que el análisis realizado se ciñe a aspectos cuantificables. De cara a trabajos posteriores, se podrían examinar los textos y las imágenes de las informaciones que infunden miedo para comprobar qué diferencias y similitudes existen en esos ámbitos.

\section{Referencias}

BARLOVENTO COMUNICACIÓN (2012): El comportamiento de la audiencia televisiva: marzo 2012: http://www.barloventocomunicacion.es/files_notas_prensa /NOTA_MARZO_2012_BARLOVENTO_COMUNICACION_AUDIENCIAS.pdf [fecha de consulta: 22 de abril de 2012].

CASTELLS, Manuel (2009): Comunicación y Poder. Madrid, Alianza.

CONDE, María Elena (1999): La experiencia de ver televisión: respuesta emocional a secuencias audiovisuales de miedo. Tesis doctoral dirigida por TORRES, Esteban. Tenerife, Universidad de La Laguna: ftp://tesis.bbtk.ull.es/ccssyhum/cs62.pdf [fecha de consulta: 28 de marzo de 2012].

GALEANO, Eduardo (2006): "El gran negocio del crimen y el miedo sacrifica la justicia”, en La Jornada (México), 4 de junio de 2006.

GIL CALVO, Enrique (2003): El miedo es el mensaje: riesgo, incerdumble y medios de comunicación. Madrid, Alianza. 
RTVE (2008): Estatuto de Información de la Corporación RTVE: http://www.rtve.es/files/70-22883-FICHERO/ESTATUTO_DE_INFORMATIVOS_MAYO2008.pdf [fecha de consulta: 21 de abril de 2012].

SOENGAS, Xosé (2007): “El control de la información en televisión”, en Ámbitos. Revista Internacional de Comunicación, $\mathrm{n}^{\circ}$ 16, Sevilla, pp. 325-341. 